\title{
DOCUMENTATION AND CULTURAL HERITAGE INVENTORIES CASE OF THE HISTORIC CITY OF AHMADABAD
}

\author{
K. Shah
}

Conservation Architect, Ahmedabad, India, khushishah@yahoo.com

KEY WORDS: Survey, Mapping, Heritage Inventories, Challenges, Data Processing, GIS Applications, Heritage management

\begin{abstract}
:
Located in the western Indian state of Gujarat, the historic city of Ahmadabad is renowned for the unparalleled richness of its monumental architecture, traditional house form, community based settlement patterns, city structure, crafts and mercantile culture. This paper describes the process followed for documentation and development of comprehensive Heritage Inventories for the historic city with an aim of illustrating the Outstanding Universal Values of its Architectural and Urban Heritage. The exercise undertaken between 2011 \& 2014 as part of the preparation of world heritage nomination dossier included thorough archival research, field surveys, mapping and preparation of inventories using a combination of traditional data procurement and presentation tools as well as creation of advanced digital database using GIS. The major challenges encountered were: need to adapt documentation methodology and survey formats to field conditions, changing and ever widening scope of work, corresponding changes in time frame, management of large quantities of data generated during the process along with difficulties in correlating existing databases procured from the local authority in varying formats. While the end result satisfied the primary aim, the full potential of Heritage Inventory as a protection and management tool will only be realised after its acceptance as the statutory list and its integration within the larger urban development plan to guide conservation, development and management strategy for the city. The rather detailed description of evolution of documentation process and the complexities involved is presented to understand the relevance of methods used in Ahmadabad and guide similar future efforts in the field.
\end{abstract}

\section{INTRODUCTION}

The historic city of Ahmadabad was founded in 1411 A.D, as the capital of independent Gujarat Sultanate. Its location on the eastern banks of river Sabarmati, along an important trade route fulfilled the requisite condition for growth of a thriving urban centre with a significant population of merchants and artisans.

Ahmadabad commands an important place amongst medieval Indian cities for its rich architectural and urban heritage. During the Sultanate era, a unique synthesis of the Islamic cultural traditions of its founders and the indigenous traditions of its majority Jain and Hindu inhabitants, led to the development of the highly acclaimed Gujarat Sultanate style renowned for the wealth of its monuments and excellence in stone craftsmanship. The urban structure of the city with its major monuments, fortifications, street networks and residential settlements was well developed by the end of late $16^{\text {th }} \mathrm{C}$. Successive periods of political dominion under the Mughal, Maratha and British rules, built upon the initial footprints of the Sultanate era, adding new institutions, adapting old ones, bringing in new materials, techniques and architectural idioms in the natural processes of cultural exchange, shared influences and evolution resulting in a stratified urban landscape of exceptional cultural significance.

Ahmadabad's walled city houses a large corpus of many remarkable monuments of historic and cultural importance. They most notably consist of the remains of citadel, fortification walls, city gates, mosques, mausoleums, temples and stepwells. The city is also renowned for its traditional houses with elaborately carved wooden facades and the community based traditional settlements 'pol'. The 'pol' are characterised by a series of courtyard houses, often ranging between 50 to 100 units, with shared longitudinal walls arranged along a narrow winding street accessed by a controlled gateway and ending in a cul-de-sac. In most cases, the 'pol' also have a small community open space 'chowk', a place of worship, a bird feeder 'chabutaro' and a community well making it a self sufficient and secure settlement unit. Several of these 'pol' came together to form 'pur'- a larger settlement unit with its boundaries defined by primary 'bazaar' market streets having its own community institutions shared by the group of $\mathrm{pol}$ and forming a self sufficient unit at the larger scale. In a natural progression of this hierarchy the city was formed by grouping of 'pur' coming together within the confines of the city walls and its larger institutions catering to the needs of entire population, creating an urban system of exceptional homogeneity.

The search for excellence in craftsmanship and cultural expression as seen in the case of monuments was also extended to the design of individual houses. The traditional wooden facades with elaborate carvings of symbols and motifs expressing the cultural beliefs of the owners are important attributes of cultural and artistic heritage. The wooden constructions with central courtyards, underground rain water harvesting cistern 'tanka' designed to provide water through the summer months of scarcity and the house form with upper floors projecting along the narrow street for mutual shading exhibits an intelligent architectural and urban design response to the hot dry climate. The traditional construction using the technique of partial timber framing and timber laced brick masonry on the ground floor along with light weight wooden façade on upper floors have exhibited resistance to earthquake forces in past and is an important attribute of its scientific value.

While the decorative idiom and building materials changed in conjunction with cultural influences and advancement in technology, the organising principles of urban and architectural form and building typology in Ahmadabad remained constant till the $20^{\text {th }} \mathrm{C}$. This continuity is reflective of their relevance to the environmental and socio-cultural context of Ahmadabad and an important attribute of its authenticity. 


\section{Background of the World Heritage Nomination:}

For almost 5 centuries after its foundation, the historic city, confined within its walls persisted in its original form and function, gracefully absorbing the gradual impacts of development and change. In the second half of $19^{\text {th }} \mathrm{C}$, industrialization, introduction of vehicular transport (cars) and development of suburbs initiated processes of structural, social and demographic changes within and around the historic city.

The need for conservation and valorisation of Cultural heritage of Ahmadabad has been emphasised by historians, archaeologists, planners and architects since $19^{\text {th }}$ C. Considerable work has been undertaken for conservation in last few decades, but, the efforts have not achieved desired results due to piecemeal nature of actions, weak policy framework and lack of political will. Today, the historic core admeasuring 5.4 sq $\mathrm{km}$ finds itself at the centre of one of the fastest growing cities in India with an urban agglomeration of more than 300 times its size. While the city has maintained its economic importance and cultural vitality, the detrimental impact of processes of change are evident as deteriorating quality of life as well as loss of its architectural and urban heritage.

Amidst serious and mounting concerns for the conservation of this unique architectural and urban heritage, on the eve of $600^{\text {th }}$ year of the city's foundation, Ahmedabad Municipal Corporation (AMC) initiated efforts for Inscription of the Historic City on UNESCO's World Heritage List. The process has been launched with the hope that the inscription will instil a sense of pride and foster greater appreciation of the cultural heritage which will eventually catalyse stronger political will, public interest and commitment towards its conservation. AMC appointed the Centre for Conservation Studies, CEPT University to undertake the task of preparation of Nomination Dossier. Documentation efforts were initiated immediately and the Historic City of Ahmadabad was placed on the tentative list of UNESCO in March 2011 following which detailed studies for the preparation of nomination dossier began.

\section{Documentation \& Heritage Inventories}

Documentation is the process of identification and recording of all relevant physical and functional information about cultural heritage assets with a view of formulating an appropriate strategy for its conservation and management. The complex nature of historic city necessitates detailed documentation and mapping of its cultural and natural characteristics, a thorough understanding of its urban morphology, historical evolution, physical \& functional attributes and socio-economic processes to guide the conservation and management strategy.

Heritage inventories are an important tool of cultural heritage management in urban context and contain a wide range of information on a building, precinct, site or open space. They typically include details on identification, location, boundary, physical features, function, condition, architectural description and identification of values contributing to the cultural significance of a particular cultural asset supported by photographs. Inventories recognised and accepted as statutory lists by governments are powerful tools for protection of cultural heritage and its management.

\section{DOCUMENTATION PROCESS}

The aim of the documentation process was to illustrate the OUVs of the Historic City of Ahmadabad through investigation of its architectural and urban heritage using mapping and heritage inventories as main tools. Thematic maps and Preliminary Inventories were prepared for various elements of the urban settlement ' $p o l$ ', natural features, traditional crafts \& market places while detailed inventories were prepared for residential and institutional properties as well as important elements of traditional urban settlements.

The major steps followed during the documentation and preparations of detailed Inventories were:

- Planning - review and learning from existing documentation, finalising the scope, identification of attributes and selection criteria for listing, selection of tools for documentation and defining processes

- Data acquisition through field surveys and archival research

- Data processing and analysis

- Data management including presentation, storage and dissemination

\section{An Overview of Existing Documentation on Ahmadabad}

Traditionally, the scope of heritage documentation and conservation in India has remained restricted to monuments. However, the historic city of Ahmadabad has exceptionally been a subject of numerous projects ranging from studies on monuments, traditional houses, urban precincts and the historic centre as a whole since early $20^{\text {th }} \mathrm{C}$. The first proposal for comprehensive documentation for the city of Ahmadabad was floated by Sir Patrick Geddes in 1915, who in the report of his visit to the city recommended a thorough survey based on the 'Cities and Town planning exhibitions prevalent in London ${ }^{1}$.

Other earlier efforts at documentation of the historic city are listed below:

A complete inventory of the historic monuments in and around the city carried out in late $19^{\text {th }} \mathrm{C}$ by the Archaeological survey of India (ASI) to identify and list the monuments of national importance. $^{2}$ The Gujarat State Department of Archaeology (SDA) has similarly undertaken the task of listing monuments of regional importance. ${ }^{3}$

In 1914, Patrick Geddes visited Ahmedabad to study the city walls demolition scheme and prepared a report in favour of their retention (Geddes, 1915). While his recommendations were not heeded to, the report is an important document as the only complete description of the now destroyed city walls and their context during the $20^{\text {th }} \mathrm{C}$.

The special census report on Ahmedabad published in 1962 identified some 500 wooden houses of exceptional architectural

1 The content and design of exhibition as proposed by Patrick Geddes can indeed be seen as the precursor of the Historic Urban Landscape approach

2 ASI's statutory list of monuments of national important protects a total of 53 structures in Ahmedabad of which 28 are located within the historic city.

${ }^{3}$ The SDA has 3 listed monuments of regional importance in Ahmedabad of which 1 is located within the historic city. 
significance. The list with details of location, ownership, age and description of listed buildings is a very valuable inventory of wooden structures of the historic city.

In 1988, a study funded by ford foundation and carried out in cooperation with the AMC identified some 250 remarkable houses and proposed recommendations for their conservation.

The library at School of Architecture and Planning at CEPT University houses a large corpus of studies on the historic city ranging from documentation of historic monuments, traditional pol houses, settlements and public places to the entire city undertaken by students and faculty members since the inception of school in 1960s.

Under the Indo-French cooperation project for the revitalisation of the walled city of Ahmedabad ${ }^{4}$ large scale survey and documentation exercise on the built heritage of city was undertaken between 1999 and 2001. This resulted in mapping of land use and building heights for almost entire walled city as well as preparation of a preliminary inventory of some 12,500 properties worthy of conservation. This inventory was the first one to be proposed as a statutory list for protection by the local government and set an important legal precedent. Unfortunately, by the time it was notified as the statutory list in 2007, a large number of identified buildings had undergone considerable modifications. Further, lack of comprehensive photographic documentation and detailed building descriptions made the implementation of its provisions difficult and the list was reduced to a reference document. While being a commendable effort, the project suffered due to lack of effective material, knowledge and technology transfer between the partners. Digitisation of original cadastral maps and the transfer of survey findings on a GIS database using MapInfo were undertaken by specialised agencies in France, but at the end of the partnership project, AMC neither had the requisite expertise or software to manage and use the data a large amount of which is untraceable today. Being one of the most recent and similar in scope, the work done by the Indo-French project could have provided valuable base data for the present exercise. Unfortunately, the considerable variations observed between the list and site condition, lack of final updated maps and absence of photographic records made it difficult to utilise the available information and update it.

Thus, in spite of a wealth of information on the city available in each of earlier project, it was necessary to undertake a fresh comprehensive documentation of the city as per the requirements of world heritage nomination dossier format.

\section{World Heritage Criteria and identification of attributes for listing:}

The nomination dossier for the city has identifies criteria, ii, v \& $\mathrm{vi}$, as being most relevant for its inscription to the World Heritage list. The significant attributes of its OUVs justifying the nomination under these criteria can be listed as:

Criteria (ii): 'Exhibit an important interchange of human values' - the monumental architecture of Gujarat Sultanate and the traditional settlement structure and pattern resulting from the

4 The cooperation project was undertaken in partnership between the Embassy of France in India \& AMC between the years $1997 \& 2006$. It also resulted in drafting of recommendations for conservation and execution of pilot conservation projects between $2003 \& 2006$. synthesis of the Islamic culture of city's founder and the culture of its majority Jain \& Hindu inhabitants.

Criteria (v): 'outstanding example of human settlement' The urban form of Ahmadabad resulting from a hierarchical organisation of house, pol, pur and city where each component acts as a self sufficient secure unit while being integrally linked with others has given rise to an urban system of exceptional homogeneity based on community agreements and cultural values.

Criteria (vi): 'associated with events or living traditions, with ideas, or with beliefs..... .' City's progressive outlook and philanthropic traditions of its elite supported numerous institutions of social and political reforms. It saw pioneering developments in the fields of education, labour rights and modern municipal governance; its association with leading personalities of freedom movement like Mahatma Gandhi \& Sardar Patel, who played important role in shaping city's culture and institutions in $20^{\text {th }} \mathrm{C}$. and Indian independence movement are some of the attributes justifying the criteria vi for nomination.

Based on the attributes identified above, it was decided to identify and record all structures with distinctive elements representative of sites OUVs as follows:

- All non-protected religious monuments, infrastructure elements and fragments representative of Gujarat Sultanate style.

- Important elements of public domain like the pol gate, community well, chabutaro, and open spaces along with integrated institutions within the traditional settlements.

- Buildings/ structures exhibiting traditional character in terms of structure, façade treatment, articulation, proportions of elements and spaces as well as interaction with the street and surroundings.

- Buildings/ structures which, in part or in whole, exhibit the influence of successive periods of city's history through minor variations in house form and decorative idiom while retaining the traditional contextual relationship with the public domain.

- Buildings and places associated with important social, cultural, economic and craft practices; historic events and personalities.

- Exceptional examples of traditional urban form and historic settlement patterns.

\section{Scope of documentation:}

The boundary of nominated property (core zone) for World Heritage inscription as identified in tentative list nomination included the traditional settlements located to the east of NorthSouth axis and the original extents of the citadel. The remaining areas of the city formed the Buffer Zone. The initial focus of the inventories was limited to traditional residential neighbourhoods within this boundary of the nominated property so defined.

This configuration considerably changed in 2012, when it was decided to propose the entire historic city as the nominated property leading to expansion of the study area to cover 543 ha distributed in 13 municipal wards consisting of more than 65000 cadastral parcels. 
The scope of data procurement during field surveys was restricted to external visual observations due to the overall scale of the exercise and time constraints.

\section{Documentation Tools:}

Printed base maps, digital camera and hand written inventory sheets were the basic tools used for field work.

While there are no uniformly agreed national data standards for documentation, the INTACH manual on Identification and documentation of Built Heritage in India provides guidelines for inventory of historic properties. The format suggested in this guideline along with the one used for the earlier inventory of Ahmedabad were used as reference while designing the building information sheets with relevant modifications to suit the scope and objective of the exercise. Standard architectural vocabulary was applied for description of structural elements and building configuration, whereas traditional nomenclature was often used for specific building elements to describe cultural imagery.

The Set or 97 digitised cadastral maps, last believed to have been updated in 1940s were the most reliable base maps available. The combined digitised base map available with AMC was further updated by adding the survey nos, incorporating the information on road widening, creation of new roads and reconfiguration of plots from official records or based on satellite images (Google) \& site observation as required. These updated base maps were used for data recording, analysis and presentation.

\section{Configuration of Documentation team and organisation of} Survey work:

The dossier project was headed and guided by a senior professor at CEPT University with many decades of research experience. The project and research work were coordinated by faculty members of the Masters in Architectural and Settlement Conservation (M.ASC) programme at the University who were engaged as research fellows for the project. This core team identified attributes of the OUVs and corresponding criteria for listing in addition to providing the framework for conducting site surveys, recording information and presentation.

The members of field survey teams were mostly young conservation architects trained at the post graduate M. ASC programme at CEPT Uni. which uses historic city as a laboratory for study of traditional architecture and settlements. They were hence familiar with the context and task at hand. The coordinators worked in close collaboration with the team providing training, guidance and support as and when required throughout the process. Fortnightly meetings were held with all members of team to discuss issues in data procurement or presentation and refinement of the method where necessary was undertaken. Surveys were conducted in groups of 2 to 3 team members depending on the detail of documentation required. The number of documentation teams varied between $1 \& 4$ during the entire duration of the project.

The preparation of GIS database using ESRI ArcGIS 10 was managed by a qualified GIS analyst trained at the Faculty of Geomatics, CEPT University.

\section{Data Procurement (Field work) and Processing:}

The field work was carried out in the first half of the day, preferably in early morning while the second half was spent in the office, for data processing and creation of the digital database. The work was undertaken by teams of 3 members with each person responsible for a separate task - mapping, photography \& detailed inventory in field as well as office. The responsibilities were often rotated to avoid fatigue. A team of 3 people could thoroughly document an entire pol or two in a day (approx. 100 buildings).

The information from survey maps including the corrections in the base map was recorded on AutoCAD using separate layers corresponding to each attribute of the property and site. The data from the descriptive survey sheets was entered in MS Excel sheets. The photographs were sorted pol-wise, renamed as per the ward name and survey numbers of the respective properties, inserted into the corresponding MS excel files and hyperlinked back to the image files. At the end of the survey, all the information was collated onto a common base map. Multiple maps were then generated presenting the attributes and their distribution at the ward level.

The overall documentation process can be broadly classified in 3 distinct phases with varied outputs in terms of details and quantum of data acquired as a direct result of constantly evolving scope of work and limitations of resources.

PHASE 1: An effort at thorough documentation of all structures within the nominated property

(Mid June 2011 to End July 2011)

Khadia Ward -3 , a characteristic residential settlement in the historic city was the first area chosen for detailed documentation. The primary unit of investigation was an individual property/parcel with traditional urban settlement 'pol' as the unit for mapping, recording and presentation of inventory data.

\section{The field work was divided into two components:}

1. Mapping of basic physical and functional attributes: All properties - Building heights \& Land use;

Inventoried properties - building conditions (with observation on modifications) \& heritage value;

Pol/Public Amenities - gate structures, bird feeder, community well, tree, black boards, open spaces 'chowks', 'chhini', right of way passages and street networks.

Changes in plot boundaries due to road widening, amalgamation or subdivision of properties were also recorded on maps.

\section{Identification and recording of cultural heritage} properties in detailed inventory formats:

Along with the documentation of physical and functional attributes detailed observation on building typology, construction material, section profile, modification to structure and articulation, date \& age of the building and description of interesting elements, architectural idiom and cultural significance were included in the detailed inventory formats.

Any non-quantifiable features of the buildings or their relation to the surroundings were recorded under 'Remarks'. The information was supplemented by digital photographs. The inventoried buildings were classified in 3 different categories of significance based on architectural, historic and contextual values and integrity of their architectural character. Traditional wooden houses were given a preference over houses from later period as well as the traditional houses with later modifications in modern material. 


\section{Challenges and limitations:}

1. Lack of updated base maps, missing details in maps at the edges of survey sheets as well as errors in digitised base maps available from AMC

2. Change to the urban structure in the form of demolitions, subdivision, modifications, amalgamation, and road widening requiring considerable efforts and time for updating base Maps through site observations.

3. Difficulty in obtaining photographs with optimum details and full building coverage in narrow and crowded street.

4. In spite of common training and guidelines variations and inconsistencies observed between the records of building condition and heritage values as well as architectural descriptions prepared by both team. This revealed a high level of subjectivity in condition assessment, interpretation of listing criteria and classification of buildings.

The necessary streamlining of this database required substantial efforts at reformatting as well as rewriting of observations and descriptions in most case and highlighted the need for further standardisation and simplification of identification criteria in subsequent stages of work and setting up of weekly review sessions to closely follow the documentation process.

\section{General Observations:}

Apart from the identification of properties to be protected the basic data on the non-listed buildings allowed many critical observations regarding ongoing process of change, factors affecting the built environment in general and threats to the preservation of listed buildings. As far as valuable properties are concerned, most properties were selected either for their Architectural and /or contextual significance based on the use of architectural idiom and its degree of intactness as evaluation criteria. Important examples of public amenities like birdfeeder $\&$ pol gate have functions in the entire pol community using both architectural and contextual significance for evaluation. There is complete lack of reliable data on age except for monuments and intuition buildings leading to minimum use of historic significance in evaluating built heritage in residential settlements.

PHASE 2: Reconnaissance survey, delineation and detailed documentation of a smaller area with high concentration of valuable properties. (June 2011 - July 2012)

While the approach adopted in the initial pilot area would have been ideal for developing a thorough understanding of the historic city with the most complete documentation of significant properties and their context, time and manpower constraints made it impossible to carry on with the same methodology.

It was evident that due to various factors of change, the concentration of valuable buildings and hence the integrity of the overall character of the neighbourhood varied from one pol to another. A more pragmatic approach by means of Identification and detailed documentation of areas with substantial amount of the traditional character retained and high concentration of valuable properties was adopted to channel the limited time and resources for their recording and conservation on a priority basis.

A primary reconnaissance (visual) survey of all the pols in the most representative historic neighbourhoods was conducted for rapid identification and mapping of potential valuable properties. Buildings with traditional characteristics expressed through material, typology, construction order, associative imagery, scale, proportion and embellishment of facade were identified and classified in 2 categories. Important institutional buildings, Religious buildings, gate structures and 'chabutaro' were also identified and located. All these information was digitally collated to a single map at the end of the survey from which a high-priority zone was roughly demarcated.

The extent of each pol was determined and the important road networks, religiously and culturally significant routes and important alleys/connections between two or more pols were highlighted.

Based on the reconnaissance surveys the further study area was delineated to contain:

- 'Pol' containing the highest concentration of valuable buildings.

- 'Pol' which retained the overall traditional urban character to a large extent was included even if they did not have exceptional buildings.

- 'Pol' containing historic or religious buildings important at city or regional level.

- Streets and areas which formed important connection between various segregated zones were identified and included for delineating a continuous area of 73 ha for detailed inventory.

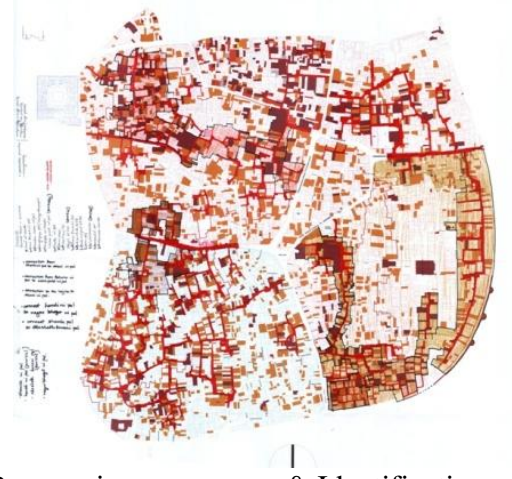

Figure 1. Reconnaissance survey \& Identification of study area

The area so defined was studied in complete details over the next year following the same documentation process as elaborated during the first phase. After a discussion between the team members and learning from earlier efforts, the selection and classification criteria simplified were further simplified to facilitate identification and avoid ambiguities. Based on the OUV and the need for providing a simple assessment guideline the attributes for identification of valuable properties were defined as below:

Homogeneity of expression of Architectural Construct expressed through: Material; Construction and associative imagery; Scale and Proportion

Facade as an aspect of Street Architecture: Facade as a generator of street space; Facade as a climatic modifier (for street as well as the house); Facade as an image of the occupant

Pol as 'Public Domain' - Tradition Vs Change: Evolving nature of public domain based on change and adaptation of newer trends of Architectural Expression while expressing continued sensitivity towards the 'street space', 'climate' and the 'image' of the occupant 
Criteria of Grading/classification of the properties were redefined Based on quantity of Embellishments and Integrity with respect to Time of Construction/architectural expression:

Highest: buildings with uniform language of articulation all over the façade with a high degree of ornamentation irrespective of the construction time period.

High: buildings with moderate or high degree of ornamentation but with articulation and structure belonging to varying time periods/expressions on the same facade.

Moderate: buildings with little or no ornamentation and modern modifications to the façade but important to overall urban character of the pol in consideration of relationship with the public domain.

The field work was conducted in the same way as earlier. Slight variation to the building information sheets were brought in to include information on building occupancy and ownership as well as approximate period of construction (in lieu of age). Data validation tool in MS Excel was used to standardise basic components of description.

\section{Challenges:}

The challenges related to the updating and errors in base map remained the same as in Phase I.

The attempt to map the ownership and occupancy details of the selected properties was unsuccessful due to reluctance for sharing correct information on the part of owners/occupiers approached during field work. Further efforts at procuring the information local tax records of AMC also failed in absence of a common denominator for identification of location and link between the two databases.

Similarly, the attempt at assigning the 'Approximate Time Period' based on the study of construction technology, type of articulation, elements on the façade and imagery in the ornamentation as opposed to age (for which no data was readily available) was only partially successful. The aim of this mapping was to identify the spatial concentration of buildings belonging to the different periods of time, and thereby understand the evolution of the city as a whole. The idea had to be abandoned due to high probability of errors in judgement as: - Articulation of structures belonging to Mughal and Maratha periods being largely similar with only minor differences made it very difficult to classify it with confidence. - Modifications, restoration and additions made to a building over the long span of its history often resulted in co-existence of elements of multiple time periods, with some structure exhibiting influences from as much as 3 time periods.

While additional verification of the building structure and interior elements might help in digging out the historical origins of the building it was beyond the scope of present documentation. Nevertheless, identification of age of buildings was retained as an important attribute of the inventory for institution buildings and was often easily traceable through archival records or architectural observation. This dating also considerably helped understand the evolution of city.

\section{Presentation of Inventories:}

The presentation of inventories and the complete documentation of residential settlements 'pol' were prepared at two different scales 1) consolidated ward database and 2) detailed pol databases called packages.

\section{Ward database packages typically contained:}

1. Key map indicating the location of the ward within the city and the list of pols selected for detailed study within the ward; Area statements of the ward and detailed study area, list of protected monuments, location of monuments, delineation and area statement for ASI zone of prohibited development and regulated development within the ward.

2. Ward level maps corresponding to various datasets: land use, building heights, valuable buildings, condition and pol amenities with area distribution on pie charts.

Pol Packages: For each pol, separate sheets were generated for the following sets of information:

1. Cover page: introductory sheet with location plan and photographs to illustrate the characteristics of the public realm of the pol and also some exemplary buildings located within the pol.

2. Map sheets: the maps representing the different sets of data viz. Land Use, Building Heights, Pol Amenities, Valuable buildings \& Conditions of Valuable Buildings and their corresponding area in the form of pie charts for each pol.

3. Inventory: the detailed data and description for each property compiled as text with a photograph of the façades in excel format.

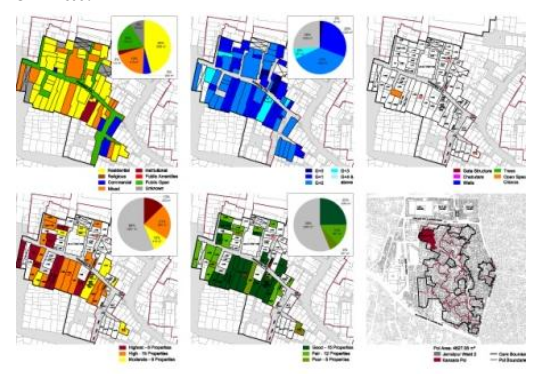

Fig. 2 - A typical presentation of pol maps

Apart from the detailed study area; detailed documentation and inventories of ASI regulated protected and regulated zone was carried out as a pilot documentation of area around the monumental complexes of the Friday mosque and royal mausoleums and a few of the city gates to evaluate the significance of cultural heritage assets located within the scope of the Ancient Monuments act.

The process created detailed inventory of some 5000 structures of varying degree of architectural significance at the end of Phase II out of which 2047 were proposed for inclusion in statutory list after further scrutiny and selection in 2013. A separate, city wide preliminary inventory of approximately 1200 institutional structures was also prepared. The decision to create separate inventory was based on variation in selection and evaluation criteria as well as the authorities wish to separate the lists for heritage conservation incentive policies. 


\section{Introduction of GIS for data management and presentation:}

At the end of $2^{\text {nd }}$ phase of documentation a large corpus of data including pol wise CAD maps, excel sheets and thousands of photographs covering more than 6000 properties made data management very cumbersome. While the pie charts provided a basic tool for analysis and understanding of the data simultaneous exploration of two or more attributes was extremely difficult with the combination of AutoCAD maps and excel database. The need for a more versatile information management system to organise, access, analyse, update and integrate spatial and non spatial attributes of inventory was acutely felt for facilitating further tasks of heritage management and preparation of conservation plan. To fulfil these requirements GIS database using ArcGIS 10 from ESRI was created for management of heritage inventories and mapping of various attributes of the site. The entire $\mathrm{CAD}$ and photographic database generated during the documentation process has been integrated in GIS and a pilot area geo-referenced with Google satellite imagery.

Jamalpur Ward 2 was identified as a pilot study area to exhibit the scope of GIS for detailed analysis and urban heritage management. Details like location and size of internal courtyard of the houses, sample data on risk and vulnerability assessment and location of public infrastructure networks have been incorporated in the GIS database of the ward for the purpose.

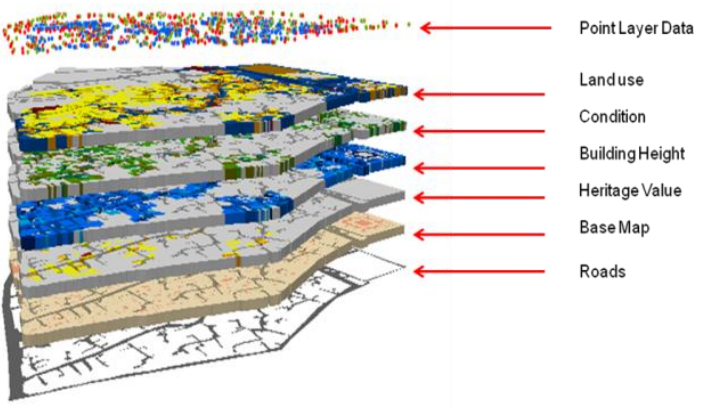

Figure 3. Data integration in GIS

Following the decision to extend the proposed boundary of nominated property to the entire historic city, AMC decided to completely scrap the old list of 2001 and notify a new list extracted from the inventories of world heritage nomination dossier. The remaining areas spread over more than half the size of the city were studied for identification and recording the valuable properties for the same

PHASE 3: Heritage Inventory of the remaining areas of the historic city. (July 2013 to August 2014)

The inventory of culturally significant properties for the remaining area posed a peculiar problem of scale. It dealt with the survey of a very large area with a low probability of finding valuable buildings. Nevertheless the study was necessary to enable a comprehensive assessment of culturally significant properties and creating a final updated list for protection.

The detailed documentation of context of listed properties which were scattered over a much larger area was out of question and a rapid survey methodology was needed. Combining the data from 2001 inventory and reconnaissance survey of 2011, base maps with probable location of valuable buildings were created beforehand and identification efforts were concentrated only in these areas with a view of economising time and efforts. Nevertheless visiting and recording scattered properties over such a large area was an extremely time consuming and tiring process. The preliminary inventory included more than 600 buildings of which only approx 430 were included in the final inventory after stringent scrutiny. Identified property were simply recorded on consolidated ward maps (CAD) and building information format in excel were generated along with updating of the GIS database.

The further detailing of the institutional inventory, its classification and grading along with preparation of building information sheets for the institutions were carried out during this stage resulting in a final inventory of 449 institutional properties proposed for listing. These include a large no. of sultanate era monuments and fragments of fortifications which were not protected in any list till now. The data was represented on a an overall city map as well as ward maps classified as per the type of institution, community patronage, height, age, condition, present and past uses, description of the building.

Apart from the inventories and comprehensive mapping of land use and building height for a large area conducted during the phase $1 \& 2$ of the process, pilot studies for disaster risk assessment, traffic management, social perception surveys and infrastructure management for the Jamalpur Ward 2. These studies offer a comprehensive understanding of values of cultural heritage as well as issues and threats to its conservation. This database will hence be instrumental in developing heritage management strategy for the historic city.

\section{STATUS OF THE INVENTORY \& WORLD HERITAGE NOMINATION:}

Following consolidation and scrutiny of data from all the 3 phases of inventories carried out between 2011 \& 2014, lists consisting of 2247 residential or mixed use properties and 449 Institutional were accepted by AMC as tentative lists of buildings to be protected by it as a local government authority. To suit the nomenclature of development plan, the buildings are classified in 4 grades - I, IIA, IIB \& III corresponding to Exceptional, highest, high \& moderate values defined in the inventories. This tentative list is presently under the process of public consultation to invite objections \& Suggestions from the members of the public. The lists shall be finalised and published in official gazette after incorporating the decisions on objections and suggestions and shall become an official list with statutory protection.

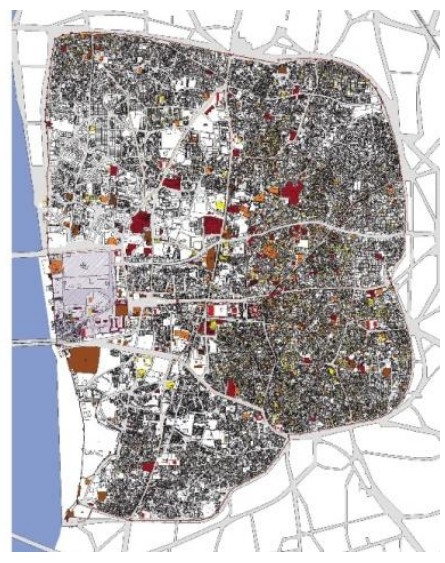

Figure 4 - Historic city of Ahmadabad with inventoried properties 
The World heritage nomination dossier is in advanced stage of preparation and the city authorities are in dialogue with the Advisory Committee on World Heritage Matters, India for its submission to UNESCO in 2016.

\section{THE WAY FORWARD}

Heritage management and monitoring change are constant processes and the regular updating of the database through incorporation of new information and documentation should be established as a practice. The inventories or lists cannot be considered as final document but must be periodically and frequently updated when new information about buildings or site is discovered.

While the cadastral map has been considerably updated by visual observations, it still is an approximation and many ambiguities need to be dealt with. This should be taken up on a priority basis using total station surveys or other advanced techniques to create reliable data support for future updating, monitoring and management activities.

The database on property ownership, occupancy, land use, vehicular ownership, and population available in varied tabular formats with different administrative departments of AMC and other Government authorities should be correlated to the spatial data in GIS for more informed and effective management.

For the listed buildings, the Building information sheets must be further updated to include detailed photographic documentation of interior features; measured drawings and condition assessment to guide conservation proposals and resources allocation.

Application of GIS database created should not be limited to use for heritage inventory or the historic city alone. Rather it must be integrated in the larger urban management systems at the level of the urban agglomeration (Ahmedabad Urban Development Authority) database to facilitate formulation of sustainable urban development strategies sympathetic to the cause of heritage conservation.

The pilot studies for vulnerabilities and risk assessment should be extended to the entire walled city and further integrated with the GIS database for risk management and cultural heritage protection.

GIS based web platform should be created for data dissemination and creating awareness regarding the significance of cultural heritage and its conservation. This tool can also be designed to allow contribution from general public to enrich the database and gather stakeholder feedbacks.

Data management should follow best practices for protection from data corruption and loss including regular backups, digital and hard copies at multiple locations.

\section{CONCLUSION}

Traditional data procurement and presentation techniques using paper based formats and mapping followed by CAD \& Excel inventories were found to be time and cost effective in creation of the data base. But they become cumbersome in updating and management of large quantities of data as seen in the case of Ahmadabad, a difficulty which can be overcome easily by integration of GIS in the documentation process at an early stage.

The documentation process achieved the primary aim of producing heritage inventories for residential and institutional structures in the historic city resulting in detailed records of more than 5000 properties representative of city's cultural heritage of which some 2696 are proposed to be accepted on the statutory list of protected buildings. It also resulted in creation of a digital database consisting of spatially linked physical and qualitative attributes data for future inventory management and a standardised output through GIS.

Major challenges like those faced due to errors in base maps should be tackled during the project inception stage to avoid revisions and ambiguities.

Preparation of heritage inventories while being a fundamental exercise in the process of establishing the significance of the world heritage sites, entail an important manpower and financial commitments on the part of local authorities and hence the database should be designed for long term use with possibilities of updating and serving larger goals in the field of urban management.

\section{ACKNOWLEDGEMENTS}

The work presented here was undertaken by the team of Centre for Conservation Studies, CEPT University, as part of the ongoing World heritage Nomination Dossier Preparation project for the Historic City of Ahmadabad, for Ahmedabad Municipal Corporation, Ahmedabad, India

\section{REFERENCES}

UNESCO, 16 November 1972. Convention Concerning the Protection of the World Cultural and Natural Heritage

UNESCO, 2013. Operational Guidelines for the Implementation of World Heritage Convention

Directorate for Architecture \& Heritage, and The French Association of Artistic Action, 2001. Recommendations for the conservation and revitalisation of the Walled City of Ahmadabad

Trivedi R.K., 1962. Wood Carving of Gujarat, Census of India, 1961, Vol.V, Gujerat, Part VII (2)

Geddes, Patrick. "Note on Ahmedabad". Environmental Design: Journal of the Islamic Environmental Design Research Centre, edited by Attilo Petruccioli. Rome: Libreria Herder, 1984.

Accessed at:

http://archnet.org/system/publications/contents/3488/original/D PC1433.pdf?1384775300

Gupta, Divay, INTACH (UK) Trust, 2007. Conservation Briefs, Identification and Documentation of Built Heritage in India 